\title{
CLINICAL USE OF AMCHA ON CERTAIN DERMATOSES
}

\author{
HITOSHI HATANO*, KAZUYA YAMAMOTO, NAMIO KONO \\ and KIICHI USUI \\ Research Project on Plasmin and Antiplasmin, \\ Department of Dermatology, School of \\ Medicine, Keio University, Tokyo \\ (Received for publication May 12, 1962)
}

Our experience in clinical use of antiplasmin drug E-amino-n-caproic acid on several kinds of skin diseases has been reported already(1-5). Recently a new antifibrinolytic substance was synthesized, and named as AMCHA(6-8). We tried to prove the effectiveness of the new antiplasmin drug AMCHA and intended to reveal the relation between plasmin activity in the blood and the effectiveness of administration of AMCHA.

\section{GENERAL METHOD OF CLINICAL EXAMINATION}

1) The clinical status of patients was carefully observed and recorded according to clinical routine in every consultation.

2) Aqueous solution of AMCHA was injected to out-patients every day as a rule. To infant patients, 2 to $5 \mathrm{ml}$ of $5 \%$ AMCHA solution was injected subcutaneously or intravenously, and to the other patients $5 \mathrm{ml}$ of the solution intravenously.

3) Relating to the clinical examination, plasmin activity in the blood was also examined by routine methods. The acceleration of whole clot lysis and euglobulin lysis and the decrease of the content of plasma fibrinogen were noticed. Appropriate and necessary controls were taken to examine the effect of AMCHA.

\section{RESULTS}

Three cases of acute dermatitis, and two cases of autosensitization dermatitis were selected for the investigation. In reproducing the degree of subjective or objective signs, the following symboles were used.

$$
\begin{aligned}
& ++\ldots \ldots \ldots \ldots \ldots \text {. Very strong } \\
& ++\ldots \ldots \ldots \text { Moderately strong }
\end{aligned}
$$

\footnotetext{
* Professor of Dermatology.
} 


\section{$+\quad \ldots \ldots \ldots \ldots$. Clearly present but weak \\ $\pm \quad \ldots \ldots \ldots \ldots$. Very slight \\ (23) Female}

Case 1.

1) Diagnosis: Acute dermatitis (on left arm).

2) Anamnesis: No significant anamnesis related to the diagnosis.

3) Case history: About one week prior to examination the lesion appeared on left arm. No previous treatment was done. Patient was seen at O.P.D. on October 23,1961 .

4) Status at the first examination: Causalgia $(++)$, red swelling $(+++)$. Acute exudative inflammation was apparently observed. Blood examination was carried out on October 23. The result showed the acceleration of euglobulin lysis, indicating high activity of plasmin.

5) Course and effect of treatment with AMCHA: Five $\mathrm{ml}$ of 5\% AMCHA solution was injected intravenously for three days from October 23 . $B y$ the first injection, exudation began to disappear. After the second injection, red swelling disappeared and causalgia was much improved; and after the third injection, it almost healed up. Blood examination was carried out 24 hours after the second injection. The result showed still accelerated euglobulin lysis.

The administration of AMCHA was interrupted for one week. On November 2, blood examination was again carried out, and the result revealed normal activity of plasmin.

6) Side effect: None.

7) External application: Boric acid and zine ointment.

8) Result: It is recognized that the administration of AMCHA was conspicuously effective and plasmin activity in blood became lower than before the administration of AMCHA.

Case 2 . (5) Female

1) Diagnosis: Autosensitization dermatitis (on the whole body).

2) Anamnesis: No significant anamnesis related to diagnosis.

3) Case history: Since about one month patient developed several nummular eczematous lesion of legs, and three days prior to examination generalized eruption appeared all over the body. Patient was seen at O.P.D. on November 24, 1961.

4) Status at the first examination: Itching $(++)$, red spots $(+)$, papules $(+)$, vesicles $(++)$, crusts $(+)$, exudation $(+)$. Blood examination was carried out on November 24. The result showed the acceleration of whole clot lysis, indicating high plasmin activity.

5) Course and effect of treatment with AMCHA: Since November 25, $5 \mathrm{ml}$ of $5 \%$ AMCHA solution was injected intravenously for eight days. After the second injection, exudation began to decrease; after the eighth injection the lesion almost healed up. Blood examination was carried out at this time, and normal activity of plasmin was noticed. 
6) Side effect: None.

7) External application: Boric acid and zinc ointment.

8) Result: It is recognized that the administration of AMCHA was effective in treating autosensitization dermatitis and plasmin activity became lower than before AMCHA treatment.

Case 3.

(20) Female

1) Diagnosis: Acute dermatitis (on face, neck and hands).

2) Anamnesis: No significant anamnesis related to the diagnosis.

3) Case history: About three days prior to examination the lesion appeared on above mentioned area. Patient was seen at O.P.D. on December 5, 1961.

4) Status at the first examination: Causalgia $(++)$, red swelling $(++)$. Acute exudative inflammation was apparently observed. Blood examination was carried out on December 7, prior to AMCHA treatment. The result revealed hypofibrinogenemia, indicating high plasmin activity in the blood.

5) Course and effect of treatment with AMCHA: Five ml of 5\% AMCHA solution was injected intravenously from December 7. After the first injection, red swelling and causalgia were much improved; after the fourth injection, the lesion almost healed up. Blood examination at this time showed normal content of plasma fibrinogen.

On December 19, blood examination was again carried out, and it revealed normal activity of plasmin.

6) Side effect: None.

7) External application: Boric acid and zinc ointment.

8) Result: It is recognized that the administration of AMCHA was effective, and that, by the administration of $A M C H A$, the course of improvement was satisfactory, and plasmin activity in blood became lower than before the treatment.

Others: One case of autosensitization dermatitis with high plasmin activity and one case of acute dermatitis with normal activity of plasmin were also treated with the same method, and the administration of AMCHA was conspicuously effective in treating these cases and suppressing plasmin activity.

\section{CONCLUSION}

Three cases of acute dermatitis, and two cases of autosensitization dermatitis were treated with 5\% AMCHA solution. The blood examination, performed before AMCHA treatment, showed high plasmin activity in 4 out of these cases, and normal plasmin activity was observed in one case of acute dermatitis.

The results of our investigations revealed that the administration of $5 \%$ AMCHA solution was effective for treating all these cases, and that plasmin activity in blood became lower than before the administration of AMCHA in 4 cases with high activity of plasmin, and no unfavorable side effect was 
observed.

\section{REFERENCES}

1. Hatano, H. et al.: Keio Igaku, 37: 353, 1960. (in Japanese)

2. Yokoyama, K. et al.: Ibid., 37:685, 1960. (in Japanese)

3. Hatano, H. et al.: Rinsho Hifu Hinyoka, 14:882, 1960. (in Japanese)

4. Hatano, H. et al.: Ibid., 15: 105, 1961 . (in Japanese)

5. Yokoyama, K. \& Hatano, H.: Keio J. Med., 8: 303, 1959.

6. Okamoto, S. \& Nagasawa, F.: U.S.A. Patent Specification, field Ser. No. 108, 245. in 1961.

7. Okamoto, S. \& Okamoto, U.: Keio J. Med., 11: 105, 1962.

8. Oshiba, S. \& Okamoto, S.: Keio J. Med., 11: 117, 1962. 